Journal of Applied Mathematics and Stochastic Analysis, 16:2 (2003), 191-200.

Printed in the USA (C) 2003 by North Atlantic Science Publishing Company

\title{
INITIAL AND BOUNDARY VALUE PROBLEMS FOR NONCONVEX VALUED MULTIVALUED FUNCTIONAL DIFFERENTIAL EQUATIONS
}

\author{
M. BENCHOHRA \\ Université de Sidi Bel Abbès, Laboratoire de Mathématiques \\ BP 89, 22000 Sidi Bel Abbès, Algérie \\ E-mail: benchohra@univ-sba.dz \\ S.K. NTOUYAS \\ University of Ioannina, Department of Mathematics \\ 45110 Ioannina, Greece \\ E-mail: sntouyas@cc.uoi.gr
}

(Received March 2002; Revised March 2003)

\begin{abstract}
In this note we investigate the existence of solutions of initial and boundary value problems defined on a compact interval to some classes of functional differential inclusions. We shall rely on a fixed point theorem for contraction multivalued maps due to Covitz and Nadler and Schafer's theorem combined with lower semicontinuous multivalued operators with decomposable values.

Keywords: Functional Differential Inclusions, Selection, Contraction Multivalued Map, Lower Semicontinuous Multivalued Map, Decomposable Valued Map, Existence, Fixed Point.
\end{abstract}

AMS (MOS) Subject Classifications: 34A60, 34K10.

\section{Introduction}

In this paper we prove existence results for the following functional differential inclusions:

$$
\left(\rho(t) y^{\prime}(t)\right)^{\prime} \in F\left(t, y_{t}\right), \text { a.e. } t \in[0, T]
$$

subject to inital

$$
y_{0}=\phi, y^{\prime}(0)=\eta
$$

or boundary conditions

$$
y_{0}=\phi, y(T)=\eta
$$

respectively, where $F: J \times C\left([-r, 0], \mathbb{R}^{n}\right) \longrightarrow \mathcal{P}\left(\mathbb{R}^{n}\right)$ is a multivalued map, $\phi \in$ $C\left([-r, 0], \mathbb{R}^{n}\right), \rho \in C\left(J, \mathbb{R}_{+}\right), \eta \in \mathbb{R}^{n}, \mathcal{P}\left(\mathbb{R}^{n}\right)$ is the family of all subsets of $\mathbb{R}^{n}$. 
For any continuous function $y$ defined on the interval $[-r, T]$ and any $t \in[0, T]$, we denote by $y_{t}$ the element of $C\left([-r, 0], \mathbb{R}^{n}\right)$ defined by

$$
y_{t}(\theta)=y(t+\theta), \quad \theta \in[-r, 0] .
$$

Here $y_{t}(\cdot)$ represents the history of the state from time $t-r$, up to the present time $t$.

Recently, in [1], the authors studied first and second order initial value problems for equation (1.1), in the case where $\rho(t)=1$, by using a fixed point theorem for contraction multivalued maps due to Covitz and Nadler [5] (see also Deimling [6]). Using a fixed point theorem for condensing multivalued maps due to Martelli, the authors have obtained an existence result for the initial value problem (1.1)-(1.2). Here, by using the fixed point theorem for contraction maps and Schaefer's theorem combined with a selestion theorem of Bressan and Colombo for lower semicontinuous multivalued operators with decomposable values, existence results are proposed for problems (1.1)(1.2) and (1.1)-(1.3).

\section{Preliminaries}

In this section, we introduce notations, definitions, and preliminary facts from multivalued analysis which are used throughout this note.

$C\left([-r, 0], \mathbb{R}^{n}\right)$ is the Banach space of all continuous functions from $[-r, 0]$ into $\mathbb{R}^{n}$ with the norm

$$
\|\phi\|=\sup \{|\phi(\theta)|:-r \leq \theta \leq 0\} .
$$

By $C\left([0, T], \mathbb{R}^{n}\right)$, we denote the Banach space of all continuous functions from $[0, T]$ into $\mathbb{R}^{n}$ with the norm

$$
\|y\|_{[0, T]}:=\sup \{|y(t)|: t \in[0, T]\} .
$$

$L^{1}\left([0, T], \mathbb{R}^{n}\right)$ denotes the Banach space of measurable functions $y:[0, T] \longrightarrow \mathbb{R}^{n}$ which are Lebesgue integrable normed by

$$
\|y\|_{L^{1}}=\int_{0}^{T}|y(t)| d t \quad \text { for all } \quad y \in L^{1}\left([0, T], \mathbb{R}^{n}\right) .
$$

$A C^{i}\left([0, T], \mathbb{R}^{n}\right)$ is the space of $i$-times differentiable functions $y:[0, T] \rightarrow \mathbb{R}^{n}$, whose $i^{\text {th }}$ derivative, $y^{(i)}$, is absolutely continuous.

Let $A$ be a subset of $[0, T] \times \mathbb{R}^{n}$. $A$ is $\mathcal{L} \otimes \mathcal{B}$ measurable if $A$ belongs to the $\sigma$-algebra generated by all sets of the form $N \times D$ where $N$ is Lebesgue measurable in $J$ and $D$ is Borel measurable in $\mathbb{R}^{n}$. A subset $B$ of $L^{1}\left([0, T], \mathbb{R}^{n}\right)$ is decomposable if, for all $u, v \in B$ and $N \subset[0, T]$ measurable, the function $u \chi_{N}+v \chi_{J-N} \in B$, where $\chi$ denotes for the characteristic function.

Let $E$ be a Banach space, $X$ a nonempty closed subset of $E$ and $G: X \rightarrow \mathcal{P}(E)$ a multivalued operator with nonempty closed values. $G$ is lower semi-continuous (l.s.c.) if the set $\{x \in X: G(x) \cap C \neq \emptyset\}$ is open for any open set $C$ in $E$. $G$ has a fixed point if there is $x \in X$ such that $x \in G(x)$.

Definition 2.1: Let $Y$ be a separable metric space and let $N: Y \rightarrow \mathcal{P}\left(L^{1}\left([0, T], \mathbb{R}^{n}\right)\right)$ be a multivalued operator. We say $N$ has property (BC) if

1) $N$ is lower semi-continuous (l.s.c.); 
2) $\quad N$ has nonempty closed and decomposable values.

Let $F:[0, T] \times C\left([-r, 0], \mathbb{R}^{n}\right) \rightarrow \mathcal{P}\left(\mathbb{R}^{n}\right)$ be a multivalued map with nonempty compact values. Assign to $F$ the multivalued operator

$$
\mathcal{F}: C\left([-r, T], \mathbb{R}^{n}\right) \rightarrow \mathcal{P}\left(L^{1}\left([0, T], \mathbb{R}^{n}\right)\right)
$$

by letting

$$
\mathcal{F}(y)=\left\{w \in L^{1}\left([0, T], \mathbb{R}^{n}\right): w(t) \in F\left(t, y_{t}\right) \text { for a.e. } t \in[0, T]\right\} .
$$

The operator $\mathcal{F}$ is called the Niemytzki operator associated with $F$. We say $F$ is of lower semi-continuous type (l.s.c. type) if its associated Niemytzki operator $\mathcal{F}$ is lower semi-continuous and has nonempty closed and decomposable values.

Next we state a selection theorem due to Bressan and Colombo.

Lemma 2.1: [3] Let $Y$ be separable metric space and let $N: Y \rightarrow \mathcal{P}\left(L^{1}\left([0, T], \mathbb{R}^{n}\right)\right)$ be a multivalued operator which has property $(B C)$. Then $N$ has a continuous selection, i.e. there exists a continuous function (single-valued) $g: Y \rightarrow L^{1}\left([0, T], \mathbb{R}^{n}\right)$ such that $g(y) \in F(y)$ for every $y \in Y$.

Let $(X, d)$ be a metric space. We use the notations:

$P(X)=\{Y \in \mathcal{P}(X): Y \neq \emptyset\}, \quad P_{c l}(X)=\{Y \in P(X): Y$ closed $\}, \quad P_{b}(X)=$ $\{Y \in P(X): Y$ bounded $\}$.

Consider $H_{d}: P(X) \times P(X) \longrightarrow \mathbb{R}_{+} \cup\{\infty\}$, given by

$$
H_{d}(A, B)=\max \left\{\sup _{a \in A} d(a, B), \sup _{b \in B} d(A, b)\right\},
$$

where $d(A, b)=\inf _{a \in A} d(a, b), d(a, B)=\inf _{b \in B} d(a, b)$.

Then $\left(P_{b, c l}(X), H_{d}\right)$ is a metric space and $\left(P_{c l}(X), H_{d}\right)$ is a generalized metric space.

Definition 2.2: A multivalued operator $N: X \rightarrow P_{c l}(X)$ is called

a) $\gamma$-Lipschitz if and only if there exists $\gamma>0$ such that

$$
H_{d}(N(x), N(y)) \leq \gamma d(x, y), \quad \text { for each } x, y \in X,
$$

b) contraction if and only if it is $\gamma$-Lipschitz with $\gamma<1$.

For more details on multivalued maps and the proof of known results cited in this section we refer to the books of Deimling [6], Górniewicz [8], Hu and Papageorgiou [9] and Tolstonogov [11].

Our considerations are based on the following fixed point theorem for contraction multivalued operators given by Covitz and Nadler in 1970 [5] (see also Deimling, [6] Theorem 11.1).

Lemma 2.2: Let $(X, d)$ be a complete metric space. If $N: X \rightarrow P_{c l}(X)$ is a contraction, then Fix $N \neq \emptyset$.

\section{Initial Value Problems}

Now, we are able to state and prove our main theorems. In this section we shall give two results for the IVP (1.1)-(1.2). Before stating and proving these results, we give the definition of a solution of the IVP (1.1)-(1.2). 
Definition 3.1: A function $y:[-r, T] \longrightarrow E$ is called solution for the IVP (1.1)(1.2) if $y(\cdot), \rho(\cdot) y(\cdot) \in C\left([-r, T], \mathbb{R}^{n}\right) \cap A C^{1}\left([0, T], \mathbb{R}^{n}\right)$ and satisfies the differential inclusion (1.1) a.e. on $[0, T]$ and the conditions (1.2).

Theorem 3.1: Assume that:

(H1) $F:[0, T] \times C\left([-r, 0], \mathbb{R}^{n}\right) \longrightarrow P_{c l}\left(\mathbb{R}^{n}\right)$ has the property that $F(\cdot, u):[0, T] \rightarrow$ $P_{c l}\left(\mathbb{R}^{n}\right)$ is measurable for each $u \in C\left([-r, 0], \mathbb{R}^{n}\right)$;

(H2) $H_{d}(F(t, u), F(t, \bar{u})) \leq l(t)\|u-\bar{u}\|$, for each $t \in[0, T]$ and $u, \bar{u} \in C\left([-r, 0], \mathbb{R}^{n}\right)$, where $l \in L^{1}([0, T], \mathbb{R})$, and $d(0, F(t, 0)) \leq l(t)$ for a.e. $t \in[0, T]$.

Then the IVP (1.1)-(1.2) has at least one solution on $[-r, T]$ provided $T \rho_{0}^{-1} \int_{0}^{T} l(s) d s<$ 1 , where $\rho_{0}=\min \{\rho(t): t \in[0, T]\}$.

Proof: Transform the problem into a fixed point problem. Consider the multivalued operator $N: C\left([-r, T], \mathbb{R}^{n}\right) \rightarrow \mathcal{P}\left(C\left([-r, T], \mathbb{R}^{n}\right)\right)$ defined by:

$N(y)=$

$$
\left\{h \in C\left([-r, T], \mathbb{R}^{n}\right): h(t)=\left\{\begin{array}{ll}
\phi(t), & \text { if } t \in[-r, 0] \\
\phi(0)+\rho(0) \eta \int_{0}^{t} \frac{d s}{\rho(s)} & \\
+\int_{0}^{t} \frac{1}{\rho(s)} \int_{0}^{s} g(\tau) d \tau d s, & \text { if } t \in[0, T], g \in S_{F(y)}
\end{array}\right\}\right.
$$

where

$$
S_{F(y)}=\left\{g \in L^{1}\left([0, T], \mathbb{R}^{n}\right): g(t) \in F\left(t, y_{t}\right) \text { for a.e. } t \in[0, T]\right\} .
$$

\section{Remark 3.1:}

(i) It is clear that the fixed points of $N$ are solutions to (1.1)-(1.2).

(ii) for each $y \in C\left([-r, T], \mathbb{R}^{n}\right)$ the set $S_{F(y)}$ is nonempty since by (H1) $F$ has a measurable selection (see [4], Theorem III.6).

We shall show that $N$ satisfies the assumptions of Lemma 2.2. The proof will be given in two steps.

Step 1: $N(y) \in P_{c l}\left(C(-r, T], \mathbb{R}^{n}\right)$ for each $y \in C\left([-r, T], \mathbb{R}^{n}\right)$.

Indeed, let $\left(y_{n}\right)_{n \geq 0} \in N(y)$ such that $y_{n} \longrightarrow \tilde{y}$ in $\left.C[-r, T], \mathbb{R}^{n}\right)$. Then $\tilde{y} \in$ $\left.C[-r, T], \mathbb{R}^{n}\right)$ and

$$
y_{n}(t) \in \phi(0)+\rho(0) \eta \int_{0}^{t} \frac{d s}{\rho(s)}+\int_{0}^{t} \frac{1}{\rho(s)} \int_{0}^{s} F\left(\tau, y_{\tau}\right) d \tau d s, \quad t \in[0, T] .
$$

Using the fact that $F$ has closed values and from the second part of (H2), we can easily show that $\int_{0}^{t} F\left(s, y_{s}\right) d s$ is closed for each $t \in[0, T]$. Then

$$
y_{n}(t) \longrightarrow \phi(0)+\rho(0) \eta \int_{0}^{t} \frac{d s}{\rho(s)}+\int_{0}^{t} \frac{1}{\rho(s)} \int_{0}^{s} F\left(\tau, y_{\tau}\right) d \tau d s, \quad t \in[0, T] .
$$

So $\tilde{y} \in N(y)$. 
Step 2: $H_{d}\left(N\left(y_{1}\right), N\left(y_{2}\right)\right) \leq \gamma\left\|y_{1}-y_{2}\right\|_{[-r, T]}$ for each $\left.y_{1}, y_{2} \in C[-r, T], \mathbb{R}^{n}\right)$ (where $\gamma<1)$.

Let $y_{1}, y_{2} \in C\left([-r, T], \mathbb{R}^{n}\right)$ and $h_{1} \in N\left(y_{1}\right)$. Then there exists $g_{1}(t) \in F\left(t, y_{1 t}\right)$ such that

$$
h_{1}(t)=\phi(0)+\rho(0) \eta \int_{0}^{t} \frac{d s}{\rho(s)}+\int_{0}^{t} \frac{1}{\rho(s)} \int_{0}^{s} g_{1}(\tau) d \tau d s, \quad t \in[0, T] .
$$

From (H2) it follows that

$$
H_{d}\left(F\left(t, y_{1 t}\right), F\left(t, y_{2 t}\right)\right) \leq l(t)\left\|y_{1 t}-y_{2 t}\right\| .
$$

Hence there is $w \in F\left(t, y_{2 t}\right)$ such that

$$
\left\|g_{1}(t)-w\right\| \leq l(t)\left\|y_{1 t}-y_{2 t}\right\|, \quad t \in[0, T] .
$$

Consider $U:[0, T] \rightarrow \mathcal{P}\left(\mathbb{R}^{n}\right)$, given by

$$
U(t)=\left\{w \in \mathbb{R}^{n}:\left\|g_{1}(t)-w\right\| \leq l(t)\left\|y_{1 t}-y_{2 t}\right\|\right\} .
$$

Since the multivalued operator $V(t)=U(t) \cap F\left(t, y_{2 t}\right)$ is measurable (see Proposition III.4 in [4]), there exists $g_{2}(t)$ a measurable selection for $V$. So, $g_{2}(t) \in F\left(t, y_{2 t}\right)$ and

$$
\left\|g_{1}(t)-g_{2}(t)\right\| \leq l(t)\left\|y_{1 t}-y_{2 t}\right\|, \text { for each } t \in[0, T] .
$$

Let us define for each $t \in[0, T]$

$$
h_{2}(t)=\phi(0)+\rho(0) \eta \int_{0}^{t} \frac{d s}{\rho(s)}+\int_{0}^{t} \frac{1}{\rho(s)} \int_{0}^{s} g_{2}(\tau) d \tau d s .
$$

Then we have

$$
\begin{aligned}
\left\|h_{1}(t)-h_{2}(t)\right\| & \leq \frac{T}{\rho_{0}} \int_{0}^{t}\left\|g_{1}(s)-g_{2}(s)\right\| d s \\
& \leq \frac{T}{\rho_{0}} \int_{0}^{t} l(s)\left\|y_{1 s}-y_{2 s}\right\| d s \\
& =\frac{T}{\rho_{0}} \int_{0}^{t} l(s)\left(\sup _{-r \leq \theta \leq 0}\left|y_{1 s}(\theta)-y_{2 s}(\theta)\right|\right) d s \\
& =\frac{T}{\rho_{0}} \int_{0}^{t} l(s)\left(\sup _{-r \leq \theta \leq 0}\left|y_{1}(s+\theta)-y_{2}(s+\theta)\right|\right) d s \\
& =\frac{T}{\rho_{0}} \int_{0}^{t} l(s)\left(\sup _{s-r \leq z \leq s}\left|y_{1}(z)-y_{2}(z)\right|\right) d s \\
& \leq \frac{T}{\rho_{0}} \int_{0}^{t} l(s)\left(\sup _{-r \leq z \leq T}\left|y_{1}(z)-y_{2}(z)\right|\right) d s \\
& \leq\left(\frac{T}{\rho_{0}} \int_{0}^{T} l(s) d s\right)\left\|y_{1}-y_{2}\right\|_{[-r, T]} .
\end{aligned}
$$

Then

$$
\left\|h_{1}-h_{2}\right\|_{[-r, T]} \leq\left(\frac{T}{\rho_{0}} \int_{0}^{T} l(s) d s\right)\left\|y_{1}-y_{2}\right\|_{[-r, T]} .
$$


By the analogous relation, obtained by interchanging the roles of $y_{1}$ and $y_{2}$, it follows that

$$
H_{d}\left(N\left(y_{1}\right), N\left(y_{2}\right)\right) \leq\left(\frac{T}{\rho_{0}} \int_{0}^{T} l(s) d s\right)\left\|y_{1}-y_{2}\right\|_{[-r, T]} .
$$

So, $N$ is a contraction and thus, by Lemma 2.2 , it has a fixed point $y$, which is solution to $(1.1)-(1.2)$.

By the help of the Schaefer's theorem combined with the selection theorem of Bressan and Colombo for lower semicontinuous maps with decomposable values, we shall present an existence result for the problem (1.1)-(1.2). Before this, let us introduce the following hypotheses which are assumed hereafter:

(H3) $F:[0, T] \times C\left([-r, 0], \mathbb{R}^{n}\right) \longrightarrow \mathcal{P}\left(\mathbb{R}^{n}\right)$ is a nonempty compact valued multivalued map such that

a) $(t, u) \mapsto F(t, u)$ is $\mathcal{L} \otimes \mathcal{B}$ measurable;

b) $u \mapsto F(t, u)$ is lower semi-continuous for a.e. $t \in[0, T]$;

(H4) For each $q>0$, there exists a function $h_{q} \in L^{1}\left([0, T], \mathbb{R}^{+}\right)$such that

$$
\begin{aligned}
\|F(t, u)\|:= & \sup \{|v|: v \in F(t, u)\} \leq h_{q}(t) \\
& \text { for a.e. } t \in[0, T] \text { and } u \in C\left([-r, 0], \mathbb{R}^{n}\right) \text { with }\|u\| \leq q .
\end{aligned}
$$

In the proof of our following theorem, we will need the auxiliary result:

Lemma 3.1: [7]. Let $F:[0, T] \times C\left([-r, 0], \mathbb{R}^{n}\right) \rightarrow \mathcal{P}\left(\mathbb{R}^{n}\right)$ be a multivalued map with nonempty, compact values. Assume (H3) and $(H 4)$ hold. Then $F$ is of l.s.c. type.

Theorem 3.2: Suppose, in addition to hypotheses $(\mathrm{H} 3)$, (H4), the following also holds:

(H5) $\|F(t, u)\|:=\sup \{|v|: v \in F(t, u)\} \leq p(t) \psi(\|u\|)$ for almost all $t \in[0, T]$ and all $u \in C([-r, 0], E)$, where $p \in L^{1}\left([0, T], \mathbb{R}_{+}\right)$and $\psi: \mathbb{R}_{+} \longrightarrow(0, \infty)$ is continuous and increasing with

$$
\frac{T}{\rho_{0}} \int_{0}^{T} p(s) d s<\int_{c}^{\infty} \frac{d \tau}{\psi(\tau)},
$$

where $c=\|\phi\|+\rho(0)|\eta| \frac{T}{\rho_{0}}$.

Then the initial value problem (1.1)-(1.2) has at least one solution.

Proof: (H3) and (H4) imply by Lemma 3.1 that $F$ is of lower semi-continuous type. Then, from Lemma 2.1 , there exists a continuous function $f: C\left([0, T], \mathbb{R}^{n}\right) \rightarrow$ $L^{1}\left([0, T], \mathbb{R}^{n}\right)$ such that $f(y) \in \mathcal{F}(y)$ for all $y \in C\left([0, T], \mathbb{R}^{n}\right)$.

We consider the problem

$$
\begin{gathered}
\left(\rho(t) y^{\prime}(t)\right)^{\prime}=f(y)(t), \text { a.e. } t \in J=[0, T], \\
y_{0}=\phi, y^{\prime}(0)=\eta .
\end{gathered}
$$

Remark 3.2: If $y \in C\left([-r, T], \mathbb{R}^{n}\right)$ is a solution of the problem (3.1)-(3.2), then $y$ is a solution to the problem (1.1)-(1.2). 
Transform problem (3.1) - (3.2) into a fixed point problem. Consider the multivalued map, $\bar{N}: C\left([-r, T], \mathbb{R}^{n}\right) \longrightarrow C\left([-r, T], \mathbb{R}^{n}\right)$ defined by:

$$
\bar{N}(y)(t)= \begin{cases}\phi(t), & \text { if } t \in[-r, 0] \\ \phi(0)+\rho(0) \eta \int_{0}^{t} \frac{d s}{\rho(s)}+\int_{0}^{t} \frac{1}{\rho(s)} \int_{0}^{s} f(y)(u) d u d s, & \text { if } t \in[0, T] .\end{cases}
$$

Clearly from (H4) and the Arzela-Ascoli theorem, the multivalued operator $\bar{N}$ is continuous and completely continuous.

In order to apply Schaefer's theorem, it remains to show that the set

$$
\mathcal{E}(\bar{N}):=\left\{y \in C\left([-r, T], \mathbb{R}^{n}\right): \lambda y=\bar{N}(y), \text { for some } \lambda>1\right\}
$$

is bounded. Let $y \in \mathcal{E}(\bar{N})$. Then $\lambda y=\bar{N}(y)$ for some $\lambda>1$. Thus, for each $t \in[0, T]$

$$
y(t)=\lambda^{-1} \phi(0)+\lambda^{-1} \rho(0) \eta \int_{0}^{t} \frac{d s}{\rho(s)}+\lambda^{-1} \int_{0}^{t} \frac{1}{\rho(s)} \int_{0}^{s} f(y)(\tau) d \tau d s .
$$

This implies by (H5) that for each $t \in[0, T]$ we have

$$
|y(t)| \leq\|\phi\|+\rho(0)|\eta| \frac{T}{\rho_{0}}+\frac{T}{\rho_{0}} \int_{0}^{t} p(s) \psi\left(\left\|y_{s}\right\|\right) d s .
$$

We consider the function $\mu$ defined by

$$
\mu(t)=\sup \{|y(s)|:-r \leq s \leq t\}, \quad 0 \leq t \leq T .
$$

Let $t^{*} \in[-r, t]$ be such that $\mu(t)=\left|y\left(t^{*}\right)\right|$. If $t^{*} \in[0, T]$, by the previous inequality we have for $t \in[0, T]$

$$
\mu(t) \leq\|\phi\|+\rho(0)|\eta| \frac{T}{\rho_{0}}+\frac{T}{\rho_{0}} \int_{0}^{t} p(s) \psi(\mu(s)) d s .
$$

If $t^{*} \in[-r, 0]$, then $\mu(t)=\|\phi\|$ and the previous inequality holds.

Let us take the right-hand side of the above inequality as $v(t)$; then we have

$$
c=v(0)=\|\phi\|+\rho(0)|\eta| \frac{T}{\rho_{0}}, \quad \mu(t) \leq v(t), \quad t \in[0, T]
$$

and

$$
v^{\prime}(t)=\frac{T}{\rho_{0}} p(t) \psi(\mu(t)), \quad t \in[0, T] .
$$

Using the nondecreasing character of $\psi$, we get

$$
v^{\prime}(t) \leq \frac{T}{\rho_{0}} p(t) \psi(v(t)), \quad t \in[0, T] .
$$

This implies for each $t \in[0, T]$ that

$$
\int_{v(0)}^{v(t)} \frac{d u}{\psi(u)} \leq \frac{T}{\rho_{0}} \int_{0}^{T} p(s) d s<\int_{v(0)}^{\infty} \frac{d u}{\psi(u)} .
$$


This inequality implies that there exists a constant $b=b(T, p, \psi)$ such that $v(t) \leq b, t \in$ $[0, T]$, and hence $\mu(t) \leq b, t \in[0, T]$. Since for every $t \in[0, T],\left\|y_{t}\right\| \leq \mu(t)$, we have

$$
\|y\|_{[r, T]}:=\sup \{|y(t)|:-r \leq t \leq T\} \leq \max (b,\|\phi\|) .
$$

This shows that $\mathcal{E}(\bar{N})$ is bounded. As a consequence of Schaefer's theorem (see [10]), we deduce that $\bar{N}$ has a fixed point which is a solution of (3.1)-(3.2) and hence from Remark 3.2, a solution to the problem (1.1)-(1.2) .

\section{Boundary Value Problems}

In the next theorem we give an existence result for the BVP (1.1), (1.3).

Definition 4.1: A function $y:[-r, T] \longrightarrow \mathbb{R}^{n}$ is called solution for the BVP (1.1), (1.3) if $y(\cdot), \rho(\cdot) y(\cdot) \in C\left([-r, T], \mathbb{R}^{n}\right) \cap A C^{1}\left([0, T], \mathbb{R}^{n}\right)$ and satisfies the differential inclusion (1.1) a.e. on $[0, T]$ and the boundary condition (1.3).

Theorem 4.1: Let $F$ satisfies (H1) and (H2). Then the BVP (1.1), (1.3) has at least one solution on $[-r, T]$.

Proof: As in Theorem 3.1 we transform the problem into a fixed point problem. Consider the multivalued operator $N_{1}: C\left([-r, T], \mathbb{R}^{n}\right) \rightarrow \mathcal{P}\left(C\left([-r, T], \mathbb{R}^{n}\right)\right)$ defined by:

$$
\begin{aligned}
& N_{1}(y):= \\
& \left\{h \in C\left([-r, T], \mathbb{R}^{n}\right): h(t)=\left\{\begin{array}{ll}
\phi(t), & \text { if } t \in[-r, 0] \\
\phi(0)+\frac{\eta-\phi(0)}{\omega(T)} \omega(t) & \\
+\int_{0}^{T} G(t, s) g(s) d s, & \text { if } t \in[0, T], g \in S_{F(y)}
\end{array}\right\}\right.
\end{aligned}
$$

where $\omega(t)=\int_{0}^{t} \frac{1}{\rho(s)} d s$ and $G$ is the Green's function for the corresponding homogenuous problem which is given by the formula

$$
G(t, s)= \begin{cases}\omega(s)\left(\frac{\omega(t)}{\omega(T)}-1\right), & \text { if } 0 \leq s \leq t \leq T \\ \omega(t)\left(\frac{\omega(s)}{\omega(T)}-1\right), & \text { if } 0 \leq t \leq s \leq T\end{cases}
$$

We shall show that $N_{1}$ satisfies the assumptions of Lemma 2.1.

Using the same reasoning as in Step 1 of Theorem 3.1, we can show that $N_{1}(y) \in$ $P_{c l}\left(C(-r, T], \mathbb{R}^{n}\right)$, for each $y \in C\left([-r, T], \mathbb{R}^{n}\right)$.

$N_{1}$ is a contraction multivalued map. Indeed, let $\left.y_{1}, y_{2} \in C[-r, T], \mathbb{R}^{n}\right)$ and $h_{1} \in$ $N_{1}\left(y_{1}\right)$. Then there exists $g_{1}(t) \in F\left(t, y_{1 t}\right)$ such that

$$
h_{1}(t)=\phi(0)+\frac{\eta-\phi(0)}{\omega(T)} \omega(t)+\int_{0}^{T} G(t, s) g_{1}(s) d s, \quad t \in[0, T] .
$$

From (H2) it follows that

$$
H_{d}\left(F\left(t, y_{1 t}\right), F\left(t, y_{2 t}\right)\right) \leq l(t)\left\|y_{1 t}-y_{2 t}\right\| .
$$


Hence, there is $w \in F\left(t, y_{2 t}\right)$ such that

$$
\left\|g_{1}(t)-w\right\| \leq l(t)\left\|y_{1 t}-y_{2 t}\right\|, \quad t \in[0, T] .
$$

Consider $U:[0, T] \rightarrow \mathcal{P}\left(\mathbb{R}^{n}\right)$, given by

$$
U(t)=\left\{w \in \mathbb{R}^{n}:\left\|g_{1}(t)-w\right\| \leq l(t)\left\|y_{1 t}-y_{2 t}\right\|\right\} .
$$

Since the multivalued operator $V(t)=U(t) \cap F\left(t, y_{2 t}\right)$ is measurable (see Proposition III.4 in [4]), there exists $g_{2}(t)$ a measurable selection for $V$. So, $g_{2}(t) \in F\left(t, y_{2 t}\right)$ and

$$
\left\|g_{1}(t)-g_{2}(t)\right\| \leq l(t)\left\|y_{1 t}-y_{2 t}\right\|, \text { for each } t \in[0, T] .
$$

Let us define for each $t \in[0, T]$

$$
h_{2}(t)=\phi(0)+\frac{\eta-\phi(0)}{\omega(T)} \omega(t)+\int_{0}^{T} G(t, s) g_{2}(s) d s .
$$

Then we have

$$
\begin{aligned}
\left\|h_{1}(t)-h_{2}(t)\right\| & \leq \int_{0}^{T} G(t, s)\left\|g_{1}(s)-g_{2}(s)\right\| d s \\
& \leq \sup _{[0, T] \times[0, T]}|G(t, s)| \int_{0}^{T} l(s)\left\|y_{1 s}-y_{2 s}\right\| d s \\
& \leq\left(\sup _{[0, T] \times[0, T]}|G(t, s)| \int_{0}^{T} l(t) d t\right)\left\|y_{1}-y_{2}\right\|_{[-r, T]} .
\end{aligned}
$$

Then

$$
\left\|h_{1}-h_{2}\right\|_{[-r, T]} \leq\left(\sup _{[0, T] \times[0, T]}|G(t, s)| \int_{0}^{T} l(t) d t\right)\left\|y_{1}-y_{2}\right\|_{[-r, T]} .
$$

By the analogous relation, obtained by interchanging the roles of $y_{1}$ and $y_{2}$, it follows that

$$
H_{d}\left(N_{1}\left(y_{1}\right), N_{1}\left(y_{2}\right)\right) \leq\left(\sup _{J \times J}|G(t, s)| \int_{0}^{T} l(t) d t\right)\left\|y_{1}-y_{2}\right\|_{[-r, T]} .
$$

So, if we choose $\sup _{[0, T] \times[0, T]}|G(t, s)| \int_{0}^{T} l(t) d t<1, N_{1}$ is a contraction, and thus, by Lemma 2.2 , it has a fixed point $y$, which is solution to (1.1), (1.3).

Using the reasoning used in the proof of Theorem 3.2, we can obtain the following result where the details are left to the reader.

Theorem 4.2: Let $F$ satisfy (H3) and

(H6) there exists a function $h \in L^{1}\left([0, T], \mathbb{R}^{+}\right)$such that

$$
\|F(t, u)\|:=\sup \{|v|: v \in F(t, u)\} \leq h(t) \text { for a.e. } t \in[0, T] \text { and } u \in C\left([-r, 0], \mathbb{R}^{n}\right) .
$$

Then the BVP (1.1), (1.3) has at least one solution on $[-r, T]$. 


\section{References}

[1] Benchohra, M. and Ntouyas, S.K., Existence results for functional differential inclusions, Electron. J. Differential Equations 41 (2001), 1-8.

[2] Benchohra, M. and Ntouyas, S.K., Existence results for second order functional differential and integrodifferential inclusions in Banach spaces, Tamkang J. Math. 32:4 (2001), 315325 .

[3] Bressan, A. and Colombo, G., Extensions and selections of maps with decomposable values, Studia Math. 90 (1988), 69-86.

[4] Castaing, C. and Valadier, M., Convex Analysis and Measurable Multifunctions, Lecture Notes in Mathematics 580, Springer-Verlag, New York 1977.

[5] Covitz, H. and Nadler, Jr., S.B., Multivalued contraction mappings in generalized metric spaces, Israel J. Math. 8 (1970), 5-11.

[6] Deimling, K., Multivalued Differential Equations, Walter de Gruyter, New York 1992.

[7] Frigon, M. and Granas, A., Théorèmes d'existence pour des inclusions différentielles sans convexité, C. R. Acad. Sci. Paris, Ser. I 310 (1990), 819-822.

[8] Gorniewicz, L., Topological Fixed Point Theory of Multivalued Mappings, Mathematics and Its Applications 495, Kluwer Academic Publishers, Dordrecht 1999.

[9] Hu, S. and Papageorgiou, N., Handbook of Multivalued Analysis, Volume I: Theory, Kluwer Academic Publishers, Dordrecht 1997.

[10] Smart, D.R., Fixed Point Theorems, Cambridge Univ. Press, Cambridge 1974.

[11] Tolstonogov, A.A., Differential Inclusions in a Banach Space, Kluwer Academic Publishers, Dordrecht 2000. 


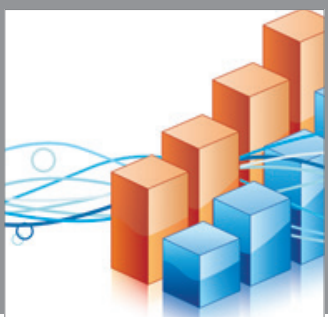

Advances in

Operations Research

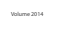

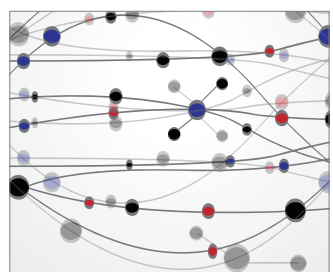

\section{The Scientific} World Journal
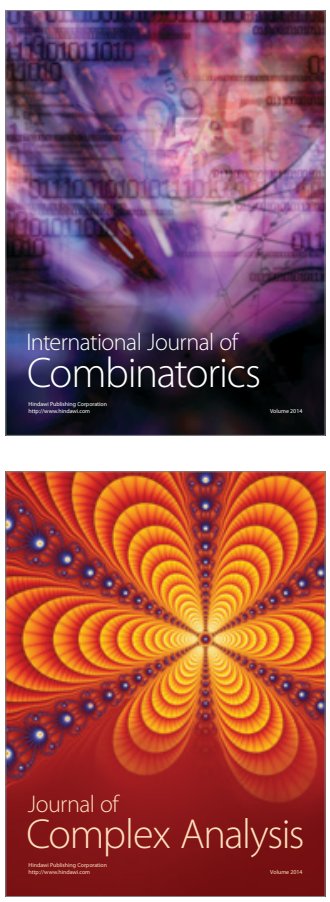

International Journal of

Mathematics and

Mathematical

Sciences
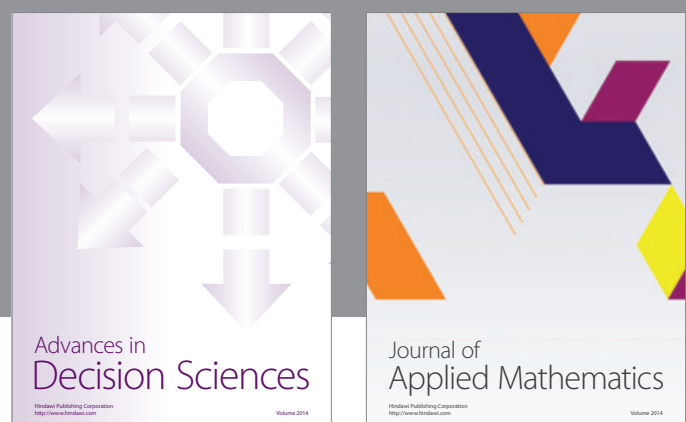

Journal of

Applied Mathematics
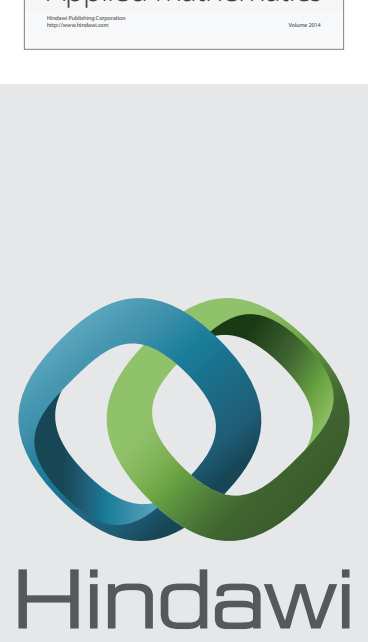

Submit your manuscripts at http://www.hindawi.com
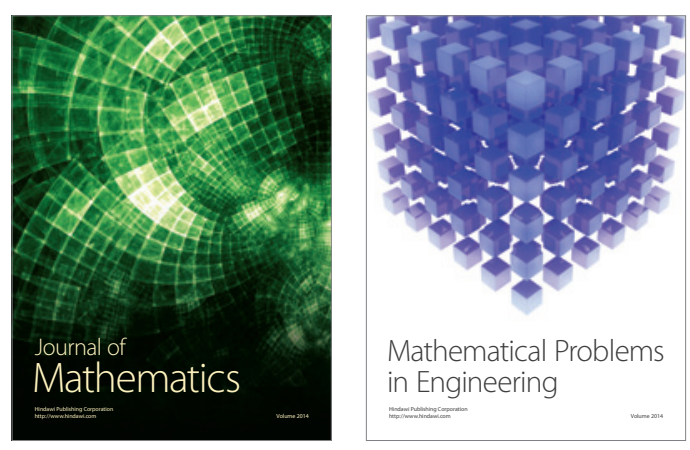

Mathematical Problems in Engineering
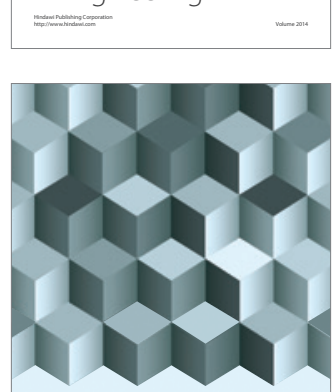

Journal of

Function Spaces
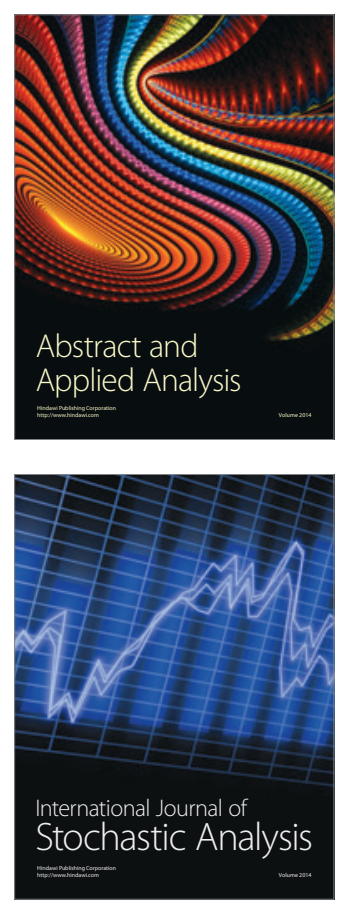

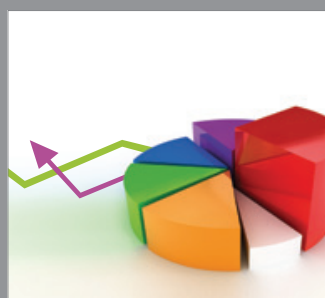

ournal of

Probability and Statistics

Promensencen
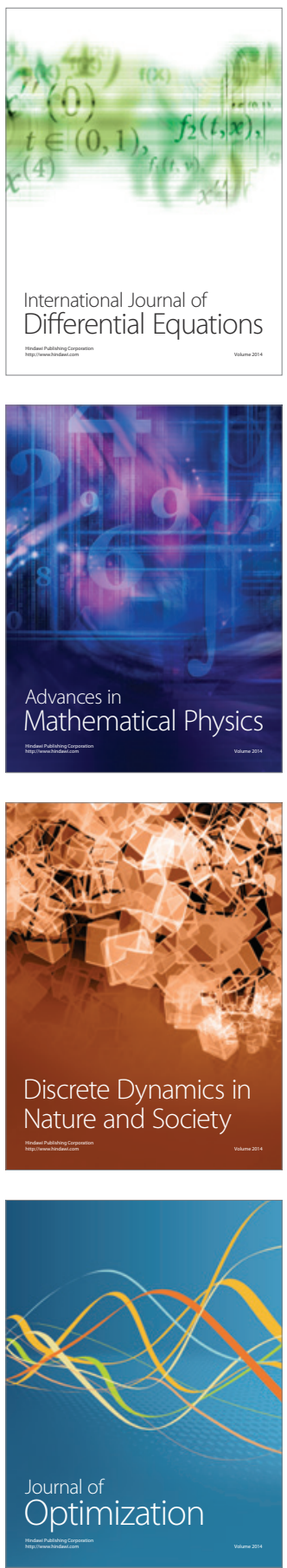